\title{
Biomarker predictors of cardiac hospitalization in chronic heart failure: a recurrent event analysis
}

\author{
Esther Vorovich, MD ${ }^{\star}$, Benjamin French, PhD $^{1,2, *}$, Bonnie Ky, MD, MSCE ${ }^{1,2}$, Lee Goldberg, \\ MD, MPH ${ }^{1}$, James C. Fang, MD $^{3}$, Nancy K. Sweitzer, MD, PhD $^{4}$, and Thomas P. Cappola, \\ MD, ScM ${ }^{1}$ \\ ${ }^{1}$ Penn Cardiovascular Institute, University of Pennsylvania Perelman School of Medicine, \\ Philadelphia, PA \\ ${ }^{2}$ Department of Biostatistics and Epidemiology, University of Pennsylvania Perelman School of \\ Medicine, Philadelphia, PA \\ ${ }^{3}$ Cardiovascular Medicine, University of Utah, Salt Lake City, UT \\ ${ }^{4}$ Cardiovascular Medicine, University of Wisconsin, Madison, WI
}

\begin{abstract}
Background-Identification of heart failure (HF) patients at risk for hospitalization may improve care and reduce costs. We evaluated nine biomarkers as predictors of cardiac hospitalization in chronic HF.
\end{abstract}

Methods and Results-In a multicenter cohort of 1512 chronic HF outpatients, we assessed the association between 9 biomarkers and cardiac hospitalization using a recurrent events approach. Over a median follow-up of 4 years, 843 participants experienced one or more hospitalizations (total 2178 hospitalizations). BNP and TnI exhibited the strongest associations with risk of hospitalization [HR 3.8 (95\% CI 2.9-4.9) and HR 3.3 (95\% CI 2.8-3.9); third vs. first tertile]. sFlt-1 exhibited the next strongest association [HR 2.8 (95\% CI 2.4-3.4)], followed by ST2 [HR 2.3 (95\% CI 2.0-2.8)] and creatinine [HR 1.9 (1.6-2.4)]. Within ischemic/nonischemic subgroups, BNP and TnI remained most strongly associated. Except for creatinine, hazard ratios for all biomarkers studied were smaller within the ischemic subgroup, suggesting greater importance of cardio-renal interactions in decompensation of ischemic HF.

Conclusion-While BNP and TnI exhibited the strongest associations with hospitalization, etiology-dependent associations for the remaining biomarkers suggest etiology-specific mechanisms for HF exacerbation. sFlt-1 exhibited a strong association with cardiac hospitalization, highlighting its potential role as a biomarker of HF morbidity.

(C) 2014 Elsevier Inc. All rights reserved.

Author for correspondence: Thomas P. Cappola, MD, ScM, 11-102 STRC, 3400 Civic Center Blvd., Philadelphia, PA 19104, Phone: 215-573-4717, Fax: 215-746-7415, thomas.cappola@uphs.upenn.edu.

Co-primary authors; equal contribution

Publisher's Disclaimer: This is a PDF file of an unedited manuscript that has been accepted for publication. As a service to our customers we are providing this early version of the manuscript. The manuscript will undergo copyediting, typesetting, and review of the resulting proof before it is published in its final citable form. Please note that during the production process errors may be discovered which could affect the content, and all legal disclaimers that apply to the journal pertain. 


\section{Introduction}

Heart failure (HF) hospitalizations are a major public health burden in the United States, accounting for over 1 million hospital admissions annually $(1,2)$. Numerous prior studies have used clinical factors and circulating biomarkers to develop predictors of adverse clinical events in HF (reviewed in (3)), the vast majority of which have focused on 'terminal' events such as ventricular assist device (VAD) placement, cardiac transplantation, and death (3). With respect to HF hospitalization, models have been developed primarily to predict time-to-first hospitalization alone or as a component of a composite terminal event (4). Although these approaches are highly relevant, they do not consider the possibly repeated nature of hospitalizations that are typical of chronic HF patients (5). As HF progresses, the highest-risk patients often experience a series of hospitalizations. These 'recurrent' events are not only burdensome to the patient but also result in a significant cost to the heath case system, accounting for more than $\$ 17$ billion in annual spending $(1,2,5)$. To address these high costs, the US Center for Medicare and Medicaid Services (CMS) and the Patient Protection and Affordable Care Act established public reporting guidelines and instituted financial penalties for centers with high rates of $\mathrm{HF}$ readmissions $(6,7)$. Improved identification of patients at high risk for hospitalization would allow for subsequent targeting of appropriate interventions thereby decreasing costs and improving patient morbidity.

As heart failure progresses, abnormalities accumulate in multiple physiologic systems. We have previously shown that assessing a panel of biomarkers that quantifies these abnormalities can improve prediction of terminal events in HF outpatients (8). Here we sought to determine the association between each of these biomarkers and risk of one or more cardiac hospitalizations. We tested the following nine biomarkers and associated pathways: high-sensitivity C-reactive protein (hsCRP) [inflammation], uric acid and myeloperoxidase (MPO) [oxidative stress], B-type natriuretic peptide (BNP) [neurohormonal activation], soluble fms-like tyrosine kinase receptor-1 (sFlt-1) and placental-like growth factor (PIGF) [vascular remodeling], troponin I (TnI) [myocyte injury], soluble toll-like receptor-2 (ST2) [myocyte stress], and creatinine [renal function] with risk of cardiac hospitalization in a multicenter cohort of 1,512 ambulatory HF patients. Our modeling approach assessed risk of one or more cardiac hospitalizations while accounting for the competing risk of terminal events. We also assessed whether biomarker associations differed according to HF etiology.

\section{Methods}

\section{Study Population}

The Penn Heart Failure Study (PHFS) is a National Heart, Lung and Blood Institutesponsored multicenter cohort study of outpatients with chronic HF recruited from referral centers at the University of Pennsylvania (Philadelphia, PA), University Hospitals/Case Medical Center (Cleveland, $\mathrm{OH}$ ), and the University of Wisconsin (Madison, WI) (8). The primary inclusion criterion is a clinical diagnosis of $\mathrm{HF}$ as determined by a $\mathrm{HF}$ specialist. The exclusion criteria are a non-cardiac condition resulting in an expected survival of less than six months as judged by the treating physician, or if patients are unwilling or unable to 
provide consent. The resultant cohort consists of outpatients with predominantly chronic systolic HF and spans a full spectrum of HF severity, ranging from mild to severe disease requiring advanced therapies. All PHFS participants provide written, informed consent. Participating institutional review boards approved the PHFS protocol.

\section{Biomarker Assays}

Biomarker levels were measured from plasma obtained at the time of study entry and banked at $-80^{\circ} \mathrm{C}$ until time of assay, as previously described (8). sFlt-1, PIGF, and MPO were measured using prototype ARCHITECT chemiluminescent microparticle-based immunoassays (Abbott Laboratories, Abbott Park, IL). BNP, TnI, hsCRP, uric acid, and creatinine were measured using standard ARCHITECT immunoassays (Abbott Laboratories, Abbott Park, IL). The TnI assay used has a lower limit detection of $5 \mathrm{ng} / \mathrm{L}$ with a coefficient of variation $\leq 10 \%$. ST2 was measured via a high-sensitivity sandwich monoclonal immunoassay (Presage ST2 Assay, Critical Diagnostics, San Diego, CA).

\section{Follow-up and Outcome Ascertainment}

PHFS participants were followed prospectively from time of study entry. Adverse events including cardiac hospitalization and the occurrence of any terminal event (all-cause mortality, cardiac transplantation, and VAD placement) were ascertained through patient contact and verified through death certificates, medical records, or contact with patients' families by research personnel. Cardiac hospitalization was defined as a hospitalization for any cardiac etiology. Research personnel, blinded to biomarker levels, adjudicated cardiac versus non-cardiac hospitalization at the time of data acquisition using source documents from the medical record.

Participants were followed until the occurrence of any terminal event or administrative censoring. If a participant was hospitalized, then follow-up continued until the occurrence of a next hospitalization, and so on, until the end of follow-up. Participants who were alive and free of cardiac transplantation or VAD placement at the end of follow-up were censored. Thus, cardiac hospitalization was treated as a recurrent event, in that participants remained at risk for hospitalization throughout follow-up, regardless of the number of hospitalizations incurred, until the occurrence of a terminal event or censoring. Participants with a VAD placement prior to study entry were excluded from the analysis.

\section{Statistical Methods}

Characteristics of study participants at enrollment were summarized using standard descriptive statistics and compared between participants with ischemic and non-ischemic HF etiology. A non-parametric, recurrent event survival curve was used to summarize the survival distribution for cardiac hospitalization (9); a standard Kaplan-Meier curve was used for the composite terminal event of all-cause death, cardiac transplantation, or VAD placement. Recurrent event models were used to determine the association between each biomarker and risk of cardiac hospitalization, which may have occurred more than once for each participant. To facilitate the comparison of associations across biomarkers, each biomarker was categorized according to tertiles of its distribution. Because hospitalization fundamentally alters the risk of death, and because hospitalization cannot occur after death, 
proper analysis of hospitalization risk requires consideration of informative censoring due to competing terminal events. A joint frailty model was used to accommodate informative censoring by all-cause death, cardiac transplantation, or VAD placement (10). The joint frailty model formulates a time-to-event model for risk of hospitalization and a time-toevent model for risk of any terminal event; an unobserved latent variable (or, frailty) is used to formulate a dependence structure between the two time-to-event models. Adjustment variables were selected a priori and included age, sex, race (Caucasian, African American, or other), and HF etiology (ischemic or non-ischemic). Additional analyses were stratified by HF etiology. In post-hoc analyses, we compared the risk of subsequent cardiac hospitalization between groups defined by biomarkers that exhibited the strongest associations in adjusted models. All analyses were completed using R 2.15.2 (R Development Core Team, Vienna, Austria), including the survrec and frailtypack extension packages (11).

\section{Results}

\section{Baseline Characteristics}

Summaries of baseline characteristics and biomarker levels of the 1512 participants in the study cohort are shown in Table 1, both in the overall cohort and according to etiology of the cardiomyopathy (ischemic and non-ischemic). The mean age of the overall cohort was 56 years (SD, 15 years), 66\% were male, $74 \%$ were Caucasian, and $22 \%$ were African American. Seventy six percent had NYHA II/III symptoms, $30 \%$ had an ischemic etiology, and the average ejection fraction (EF) was 33\%. Guideline-based therapies were highly prevalent ( $87 \%$ ACE inhibitors or ARBs, $88 \%$ beta blockers, $35 \%$ aldosterone antagonists). On average, participants with ischemic cardiomyopathy were more likely to be older, male, Caucasian, have a greater burden of cardiovascular risk factors, higher NYHA class, lower glomerular filtration rates, and lower ejection fractions than non-ischemic participants. Median levels of BNP, PIGF, SFlt-1, hsCRP, ST2, creatinine, uric acid, and percentage of participants with detectable troponin were higher in the ischemic subgroup.

\section{Cardiac Hospitalizations}

Follow-up time was a median of 4.0 years and a maximum of 8.9 years. 843 participants (55.8\%) experienced one or more cardiac hospitalizations, for a total of 2178 hospitalizations: 347 participants experienced 1 hospitalization, 193 experienced 2, 112 experienced 3, 77 experienced 4, and 114 experienced 5 or more. 669 participants (44.2\%) were never hospitalized. Median survival free from cardiac hospitalization was 3.9 years in the overall cohort (Figure 1a). 453 participants (30.0\%) experienced a terminal event of death, cardiac transplantation, or VAD placement (Figure 1b). Among participants who subsequently experienced death, cardiac transplantation, or VAD placement, median survival free from cardiac hospitalization was 2.6 years (Figure 1c). Analysis of a priori selected covariates showed that ischemic etiology was an independent predictor of hospitalization (HR 1.3, 95\% CI 1.1-1.5), as was male gender (HR 1.3, 95\% CI 1.1-1.6), African American race (HR 1.5, 95\% CI 1.3-1.8), and increasing age. 


\section{Associations between Biomarkers and Risk of Cardiac Hospitalization}

Among all participants, several biomarkers were significantly associated with risk of cardiac hospitalization (Table 2). BNP and TnI exhibited the strongest associations in both adjusted and unadjusted models. Among the remaining biomarkers, all but PIGF showed statistically significant associations with risk of cardiac hospitalization in both the adjusted and unadjusted models, albeit with smaller hazard ratios than TnI or BNP. Furthermore, all biomarkers showed a dose-response relationship, with larger hazard ratios with increasing tertile of plasma biomarker level.

The strength of association between individual biomarkers and risk of cardiac hospitalization showed interesting differences by HF etiology (Figure 2). Among participants with non-ischemic HF, the biomarkers that exhibited the strongest associations with a HR > 2 when comparing the third to first tertile were: BNP (HR 4.1), TnI (HR 3.5), sFlt-1 (HR 3.2) and ST2 (HR 2.8). These findings implicate neurohormonal activation, myocyte necrosis, vascular remodeling, and myocyte stress as precursors of cardiac hospitalization in non-ischemic HF. In the ischemic subgroup, BNP (HR 3.2) and TnI (HR 2.7) showed the strongest associations, but the magnitudes of the hazard ratios were smaller than in nonischemics. Creatinine (HR 2.7) showed the third-strongest association with cardiac hospitalization in the ischemic subgroup, which differed substantially from the association in non-ischemics (HR 1.8). This finding suggests a greater importance for cardio-renal interactions as a precursor to hospitalization in ischemic HF.

Because BNP and TnI showed the strongest associations overall and in both subgroups, we explored their combined association with cardiac hospitalization. After stratifying the overall cohort by levels of BNP (dichotomized at the median of $173 \mathrm{pg} / \mathrm{mL}$ ) and $\mathrm{TnI}$ (dichotomized as detectable versus not), risk of cardiac hospitalization increased with elevation of either BNP or TnI and further increased in participants with elevations in both (Table 3).

\section{Discussion}

While numerous prior studies have evaluated a multitude of biomarkers in HF populations, these studies have focused on the outcome of death or another terminal event such as cardiac transplantation and/or VAD placement, and have not focused on hospitalization as a primary outcome. Rather, hospitalizations were included either as a secondary outcome or as part of a composite hospitalization and terminal event outcome in a time-to-first-event analysis. This approach, while standard among clinical trials, does not take into account the multiple hospitalizations experienced by HF patients over time and so does not fully represent the patients' overall experience with their disease. Moreover, in a time-to-first analysis, all patients contribute equally to the analysis regardless of the number of hospitalizations incurred, thereby creating a disconnect between the included data and the patients' true burden of disease (5). In addition, death and hospitalization are competing risk events, because hospital admission fundamentally alters the risk of death and admission cannot occur after death (12). Heidenreich et al. showed divergent trends in rates of rehospitalization and mortality, which demonstrates the limitations of viewing hospitalization as simply a surrogate marker for mortality (13). Rising health care costs and 
increasing focus on quality performance measures further underscore the relevance of $\mathrm{HF}$ hospitalization as an important clinical endpoint (6). To our knowledge, our study is the first multi-biomarker study of chronic heart failure to evaluate hospitalization as the primary outcome, to include recurrent hospitalizations in our analyses, and to properly account for competing risks.

We tested a panel of nine biomarkers that span several biological processes and have been previously implicated in HF pathophysiology. Among these candidate biomarkers, BNP and TnI exhibited the strongest association with risk of cardiac hospitalization overall and in both the ischemic and non-ischemic subgroups. BNP and TnI have been shown to associate independently with morbidity and mortality across a wide breadth of HF severity (14-21) and to have an additive 'dose response' effect, with stronger associations seen with elevations of both biomarkers over each biomarker alone $(22,23)$. Our study extends this consistent finding to a recurrent event framework regarding risk of cardiac hospitalization.

As shown in Figure 2, nearly all biomarkers showed weaker associations in the ischemic compared to the nonischemic subgroup. Because HF is a heterogeneous disorder it seems likely that within any HF population there would be subgroups within which biomarkers provide more, or less, information about the risk of incident events. In our cohort, comparison of the ischemic versus nonischemic subgroups in Table 1 shows that ischemic HF patients have a higher prevalence of comorbid conditions such as hypertension, diabetes, and dyslipidemia. We speculate that these comorbid conditions influence biomarker levels independently of $\mathrm{HF}$ and as a result dampen associations between many biomarker levels and outcomes to a greater extent in ischemic HF.

By contrast, creatinine, a well-established marker of renal function and prognosis in HF patients $(24,25)$, exhibited a stronger association in the ischemic subgroup, suggesting a greater impact of cardiorenal interactions as a precursor to hospitalization in ischemic HF. The precise biological mechanisms for this finding are unclear. However, it is likely that intrinsic primary renal disease is more prevalent in ischemic HF due to shared etiologic factors, such as diabetes and hypertension, which contribute to both coronary artery disease and to renal dysfunction. As shown in Table 1, hypertension and diabetes were more common among patients with ischemic heart disease in our cohort, and baseline creatinine levels were on average higher. We speculate that the population of patients with HF tends to have worse intrinsic renal function and further renal embarrassment is therefore more likely and more indicative of subsequent decompensation.

We note that sFlt-1, a marker of vascular remodeling that is not commonly studied in HF populations, showed a strong association with risk of hospitalization in the overall cohort and especially so in the non-ischemic subgroup. sFlt-1 is a soluble anti-angiogenic factor that binds to and sequesters vascular endothelial growth factor (26). Profound elevations in sFlt-1 that accompany pregnancy may cause excessive impairment of angiogenesis, leading to microvascular loss in the heart and peripartum cardiomyopathy (27). Our findings support the hypothesis that excessive sFlt-1 contributes to HF decompensation, as evidenced by a higher likelihood of hospitalization. It is also noteworthy that sFlt-1 had stronger associations in both subgroups than did the clinically approved biomarker ST2, which 
assesses an IL-33 mediated stress-response pathway that affects myocardial function (28). Among the remaining biomarkers, several have been assessed extensively in HF populations and have become established markers of prognosis, including uric acid, MPO, and hsCRP (29-33). These biomarkers showed significant association with risk of hospitalization overall and in both subgroups, but the strength of these associations was substantially weaker than that for the biomarkers discussed above. This finding suggests that oxidative stress and inflammation may be less important to assess in predicting likelihood of cardiac hospitalization in chronic HF. PIGF did not exhibit a significant association after adjustment for covariates.

Our study has several relevant strengths and limitations. This was a large cohort study with participants recruited from HF referral centers with broad inclusion criteria. As a result, the study cohort represents a broad spectrum of HF disease severity, and the population is diverse with $44 \%$ female and $26 \%$ non-Caucasian. However, we acknowledge that certain subgroups, such as non-ischemic HF, may be over-represented, and others, such as the elderly, maybe under-represented. In particular, we have a very small proportion $(<8 \%)$ of patients with HF and preserved ejection fraction and are unable to assess the association between biomarkers and risk of hospitalization in this subgroup. We did not evaluate serial measures of biomarkers, which have been previously shown to associate with adverse outcomes $(14,18,34)$, and our panel included only select biomarkers that were chosen to represent a specific biologic processes. It is plausible that additional markers that were not evaluated, such as high-sensitivity troponin or galectin-3, could have stronger associations. Future discovery of relevant biomarkers using unbiased approaches such as metabolomics and proteomics may yield further advances. Finally, we focused on association models rather than on the evaluation of risk-prediction models using receiver operating characteristic curves or risk reclassification analyses. There are currently no available statistical methods to build a multi-marker risk score and evaluate its prediction accuracy for a recurrent event that may be censored by a competing terminal event.

These findings may influence clinical care by allowing clinicians to better predict HF patients at higher risk of hospitalization. Reducing HF hospitalizations and early readmissions has been a focus of recent payment reform. Whether changing HF therapy or implementing disease management strategies will influence either the levels of biomarkers or the risk of hospitalization is unknown and will require further study.

In summary, we found that while BNP and troponin exhibited the strongest association with risk of cardiac hospitalization, the strength of the associations between the remaining biomarkers varied by HF etiology. sFlt-1 exhibited a stronger association than several wellestablished biomarkers, highlighting its potential role as a biomarker for HF morbidity. Further studies are needed to determine the optimal biomarkers involved in HF disease progression, as well as to develop statistical methods to quantify prediction accuracy in the setting of recurrent events and competing risks.

\section{Acknowledgments}

None 
Funding sources: Funded by NIH R01HL088577. Funding for assays was provided by Abbott Diagnostics and Critical Diagnostics

Disclosures:

Drs. Ky and Cappola are co-inventors on a pending patent for use of sFlt-1 as a cardiac biomarker.

\section{References}

1. Gheorghiade M, Vaduganathan M, Fonarow GC, Bonow RO. Rehospitalization for heart failure: problems and perspectives. Journal of the American College of Cardiology. 2013 Jan 29; 61(4): 391-403. [PubMed: 23219302]

2. Go AS, Mozaffarian D, Roger VL, Benjamin EJ, Berry JD, Borden WB, et al. Heart disease and stroke statistics--2013 update: a report from the American Heart Association. Circulation. 2013 Jan 1; 127(1):e6-e245. [PubMed: 23239837]

3. Braunwald E. Heart Failure. JACC: Heart Failure. 2013; 1(1):1-20. [PubMed: 24621794]

4. Kociol RD, McNulty SE, Hernandez AF, Lee KL, Redfield MM, Tracy RP, et al. Markers of decongestion, dyspnea relief, and clinical outcomes among patients hospitalized with acute heart failure. Circ Heart Fail. 2013 Mar; 6(2):240-245. [PubMed: 23250981]

5. Anker SD, McMurray JJ. Time to move on from 'time-to-first': should all events be included in the analysis of clinical trials? Eur Heart J. 2012 Nov; 33(22):2764-2765. [PubMed: 22927554]

6. Desai AS, Stevenson LW. Rehospitalization for heart failure: predict or prevent? Circulation. 2012 Jul 24; 126(4):501-506. [PubMed: 22825412]

7. Bradley EH, Curry L, Horwitz LI, Sipsma H, Wang Y, Walsh MN, et al. Hospital strategies associated with 30-day readmission rates for patients with heart failure. Circulation Cardiovascular quality and outcomes. 2013 Jul; 6(4):444-450. [PubMed: 23861483]

8. Ky B, French B, Levy WC, Sweitzer NK, Fang JC, Wu AH, et al. Multiple biomarkers for risk prediction in chronic heart failure. Circ Heart Fail. 2012 Mar 1; 5(2):183-190. [PubMed: 22361079]

9. Peña EASR, Hollander M. Nonparametric estimation with recurrent event data. Journal of the American Statistical Association. 2001; (96):1299-1315.

10. Rondeau VM-PS, Jacqmin-Gadda H, Brouste V, Soubeyran P. Joint frailty models for recurring events and death using maximum penalized likelihood estimation: Application on cancer events. Biostatistics. 2007; 8:708-721. [PubMed: 17267392]

11. Rondeau VMY, Gonzalez JR. Frailtypack: An R package for the analysis of correlated survival data with frailty models using penalized likelihood estimation or parametrical estimation. Journal of Statistical Software. 2012; 47:1-28.

12. Lau B, Cole SR, Gange SJ. Competing risk regression models for epidemiologic data. Am J Epidemiol. 2009 Jul 15; 170(2):244-256. [PubMed: 19494242]

13. Heidenreich PA, Sahay A, Kapoor JR, Pham MX, Massie B. Divergent trends in survival and readmission following a hospitalization for heart failure in the Veterans Affairs health care system 2002 to 2006. Journal of the American College of Cardiology. 2010 Jul 27; 56(5):362-368. [PubMed: 20650356]

14. Anand IS, Fisher LD, Chiang YT, Latini R, Masson S, Maggioni AP, et al. Changes in brain natriuretic peptide and norepinephrine over time and mortality and morbidity in the Valsartan Heart Failure Trial (Val-HeFT). Circulation. 2003 Mar 11; 107(9):1278-1283. [PubMed: 12628948]

15. Fonarow GC, Peacock WF, Phillips CO, Givertz MM, Lopatin M, Committee ASA, et al. Admission B-type natriuretic peptide levels and in-hospital mortality in acute decompensated heart failure. Journal of the American College of Cardiology. 2007 May 15; 49(19):1943-1950. [PubMed: 17498579]

16. Hudson MP, O'Connor CM, Gattis WA, Tasissa G, Hasselblad V, Holleman CM, et al. Implications of elevated cardiac troponin $\mathrm{T}$ in ambulatory patients with heart failure: a prospective analysis. Am Heart J. 2004 Mar; 147(3):546-552. [PubMed: 14999208] 
17. Latini R, Masson S, Anand IS, Missov E, Carlson M, Vago T, et al. Prognostic value of very low plasma concentrations of troponin $\mathrm{T}$ in patients with stable chronic heart failure. Circulation. 2007 Sep 11; 116(11):1242-1249. [PubMed: 17698733]

18. Miller WL, Hartman KA, Burritt MF, Grill DE, Jaffe AS. Profiles of serial changes in cardiac troponin $\mathrm{T}$ concentrations and outcome in ambulatory patients with chronic heart failure. Journal of the American College of Cardiology. 2009 Oct 27; 54(18):1715-1721. [PubMed: 19850213]

19. Parissis JT, Ikonomidis I, Rafouli-Stergiou P, Mebazaa A, Delgado J, Farmakis D, et al. Clinical characteristics and predictors of in-hospital mortality in acute heart failure with preserved left ventricular ejection fraction. Am J Cardiol. 2011 Jan; 107(1):79-84. [PubMed: 21146691]

20. Perna ER, Macin SM, Canella JP, Augier N, Stival JL, Cialzeta JR, et al. Ongoing myocardial injury in stable severe heart failure: value of cardiac troponin T monitoring for high-risk patient identification. Circulation. 2004 Oct 19; 110(16):2376-2382. [PubMed: 15477403]

21. van Veldhuisen DJ, Linssen GC, Jaarsma T, van Gilst WH, Hoes AW, Tijssen JG, et al. B-type natriuretic peptide and prognosis in heart failure patients with preserved and reduced ejection fraction. Journal of the American College of Cardiology. 2013 Apr 9; 61(14):1498-1506. [PubMed: 23500300]

22. Horwich TB, Patel J, MacLellan WR, Fonarow GC. Cardiac troponin I is associated with impaired hemodynamics, progressive left ventricular dysfunction, and increased mortality rates in advanced heart failure. Circulation. 2003 Aug 19; 108(7):833-838. [PubMed: 12912820]

23. Miller WL, Hartman KA, Burritt MF, Grill DE, Rodeheffer RJ, Burnett JC Jr, et al. Serial biomarker measurements in ambulatory patients with chronic heart failure: the importance of change over time. Circulation. 2007 Jul 17; 116(3):249-257. [PubMed: 17592074]

24. Dries DL, Exner DV, Domanski MJ, Greenberg B, Stevenson LW. The prognostic implications of renal insufficiency in asymptomatic and symptomatic patients with left ventricular systolic dysfunction. Journal of the American College of Cardiology. 2000 Mar 1; 35(3):681-689. [PubMed: 10716471]

25. Hillege HL, Nitsch D, Pfeffer MA, Swedberg K, McMurray JJ, Yusuf S, et al. Renal function as a predictor of outcome in a broad spectrum of patients with heart failure. Circulation. 2006 Feb 7; 113(5):671-678. [PubMed: 16461840]

26. Ky B, French B, Ruparel K, Sweitzer NK, Fang JC, Levy WC, et al. The vascular marker soluble fms-like tyrosine kinase 1 is associated with disease severity and adverse outcomes in chronic heart failure. Journal of the American College of Cardiology. 2011 Jul 19; 58(4):386-394. [PubMed: 21757116]

27. Patten IS, Rana S, Shahul S, Rowe GC, Jang C, Liu L, et al. Cardiac angiogenic imbalance leads to peripartum cardiomyopathy. Nature. 2012 May 17; 485(7398):333-338. [PubMed: 22596155]

28. Sanada S, Hakuno D, Higgins LJ, Schreiter ER, McKenzie AN, Lee RT. IL-33 and ST2 comprise a critical biomechanically induced and cardioprotective signaling system. J Clin Invest. 2007 Jun; 117(6):1538-1549. [PubMed: 17492053]

29. Tang WH, Shrestha K, Troughton RW, Borowski AG, Klein AL. Integrating plasma highsensitivity C-reactive protein and myeloperoxidase for risk prediction in chronic systolic heart failure. Congest Heart Fail. 2011 May-Jun;17(3):105-109. [PubMed: 21609382]

30. Tang WH, Tong W, Troughton RW, Martin MG, Shrestha K, Borowski A, et al. Prognostic value and echocardiographic determinants of plasma myeloperoxidase levels in chronic heart failure. Journal of the American College of Cardiology. 2007 Jun 19; 49(24):2364-2370. [PubMed: 17572253]

31. Wu AH, Ghali JK, Neuberg GW, O'Connor CM, Carson PE, Levy WC. Uric acid level and allopurinol use as risk markers of mortality and morbidity in systolic heart failure. Am Heart J. 2010 Nov; 160(5):928-933. [PubMed: 21095282]

32. Yin WH, Chen JW, Jen HL, Chiang MC, Huang WP, Feng AN, et al. Independent prognostic value of elevated high-sensitivity C-reactive protein in chronic heart failure. Am Heart J. 2004 May; 147(5):931-938. [PubMed: 15131554]

33. Anker SD, Doehner W, Rauchhaus M, Sharma R, Francis D, Knosalla C, et al. Uric acid and survival in chronic heart failure: validation and application in metabolic, functional, and hemodynamic staging. Circulation. 2003 Apr 22; 107(15):1991-1997. [PubMed: 12707250] 
34. Ky B, French B, McCloskey K, Rame JE, McIntosh E, Shahi P, et al. High-sensitivity ST2 for prediction of adverse outcomes in chronic heart failure. Circ Heart Fail. 2011 Mar; 4(2):180-187. [PubMed: 21178018] 


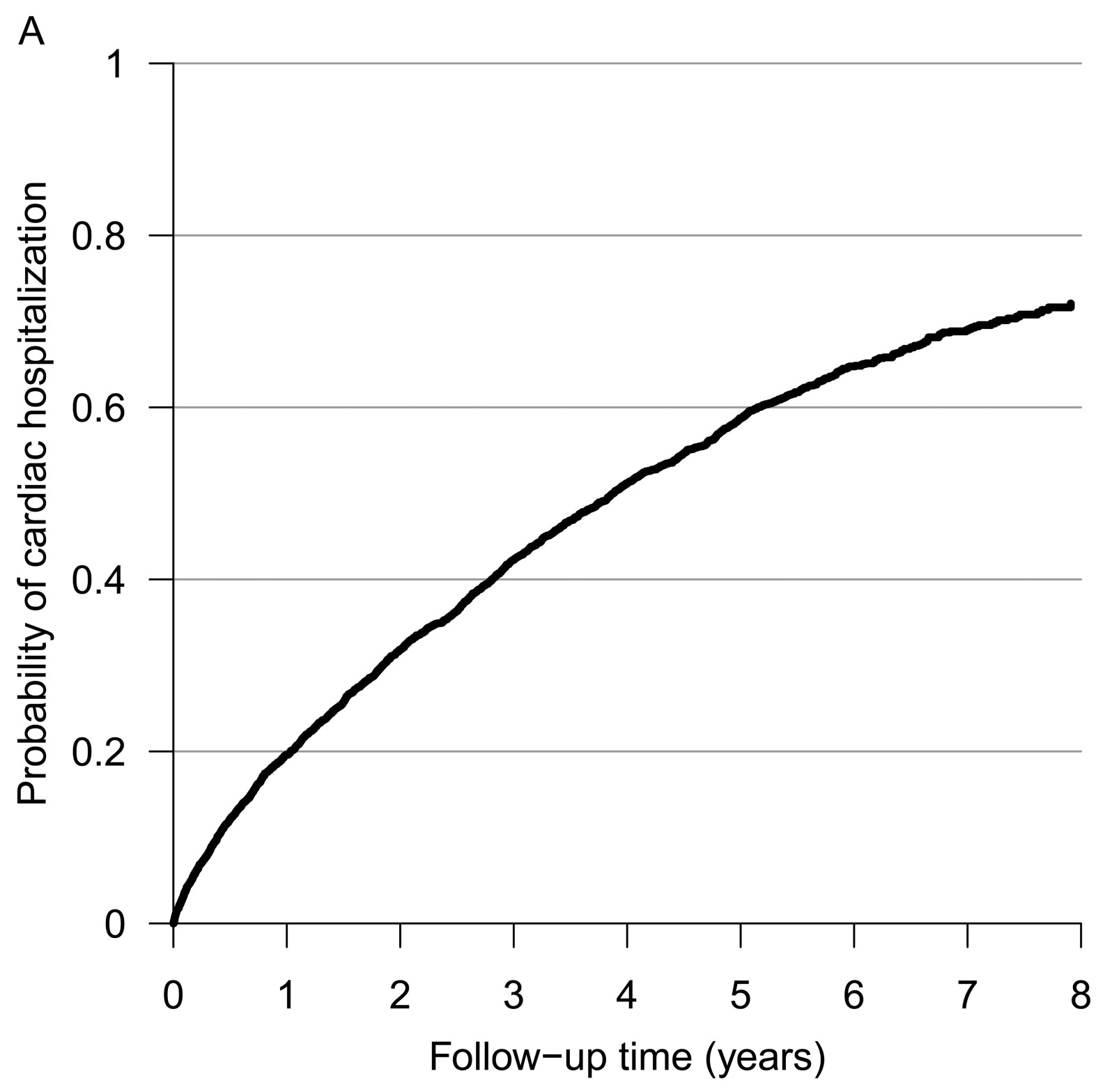

Number at risk

$\begin{array}{lllllllll}1512 & 1331 & 1233 & 1106 & 766 & 558 & 396 & 170 & 39\end{array}$ 


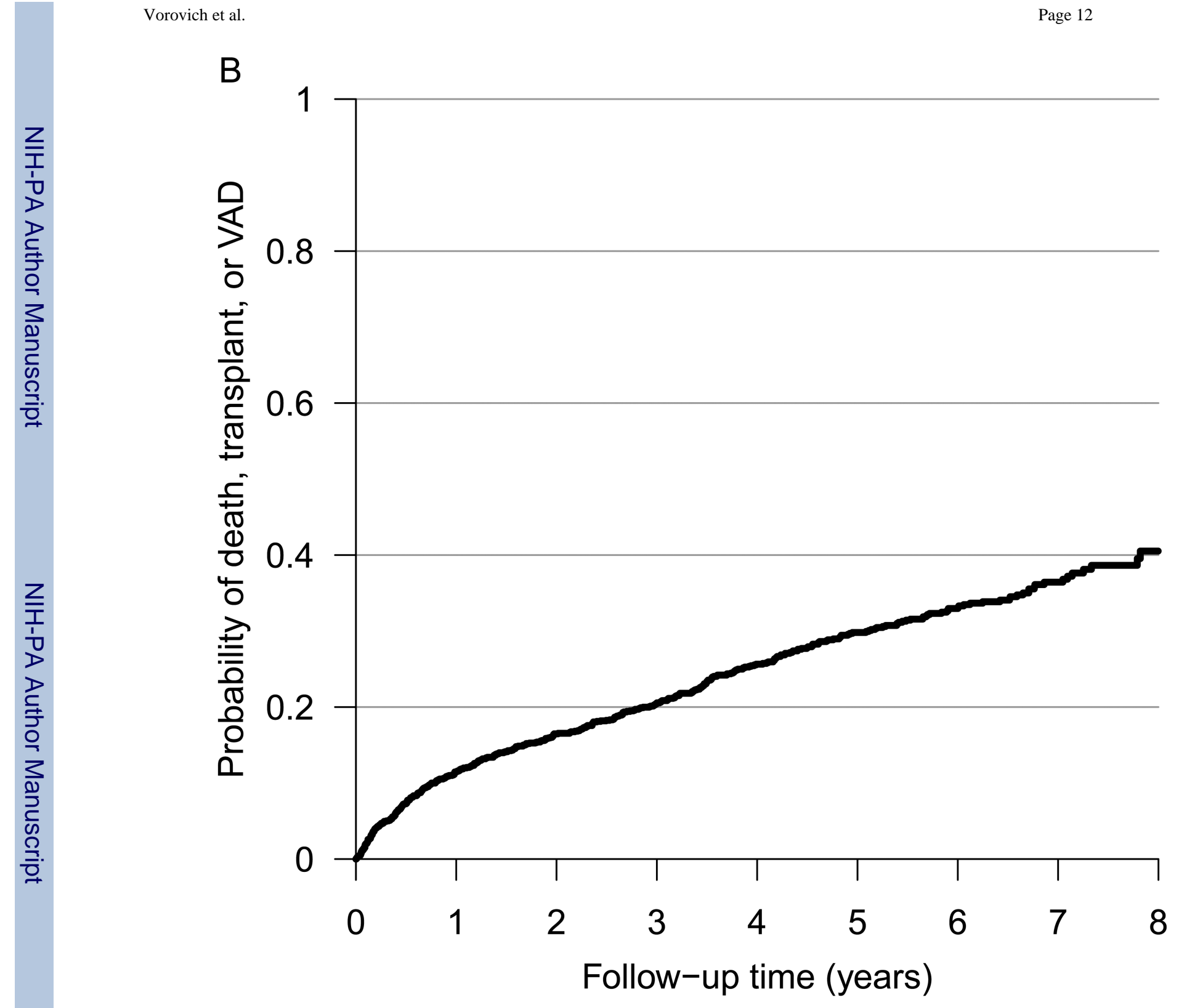

Number at risk

$\begin{array}{lllllllll}1512 & 1331 & 1233 & 1106 & 766 & 558 & 396 & 170 & 39\end{array}$ 


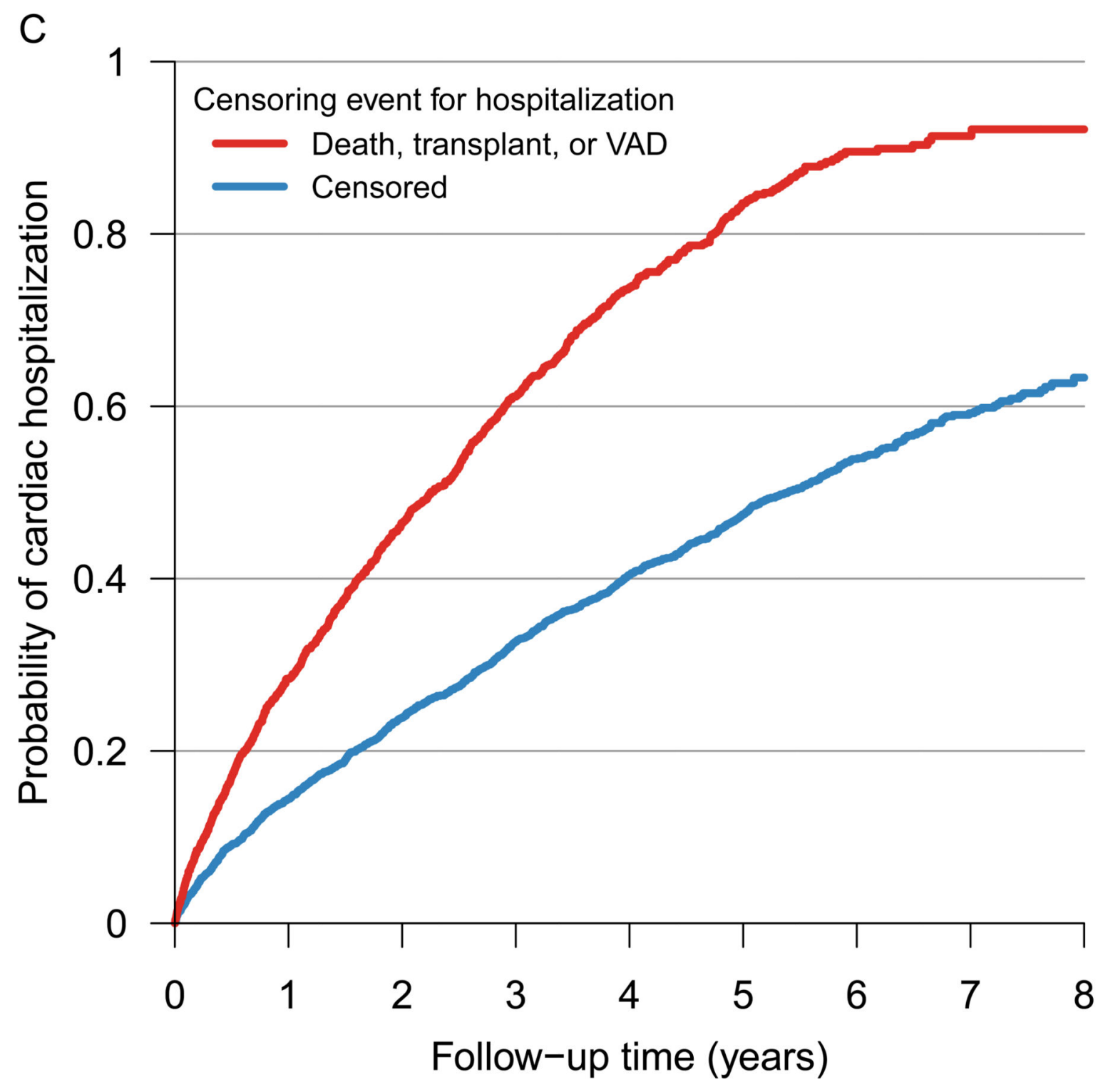

Number at risk

$\begin{array}{ccccccccc}-453 & 280 & 205 & 147 & 83 & 45 & 23 & 8 & 1 \\ -1059 & 1051 & 1028 & 959 & 683 & 513 & 373 & 162 & 38\end{array}$

Figure 1.

Probability of incident cardiac hospitalization for all participants (A) and probability of allcause death, cardiac transplantation, or VAD placement for all participants (B). Probability of incident cardiac hospitalization stratified by the subsequent occurrence of all-cause death, cardiac transplantation, or VAD placement during follow-up (C). 

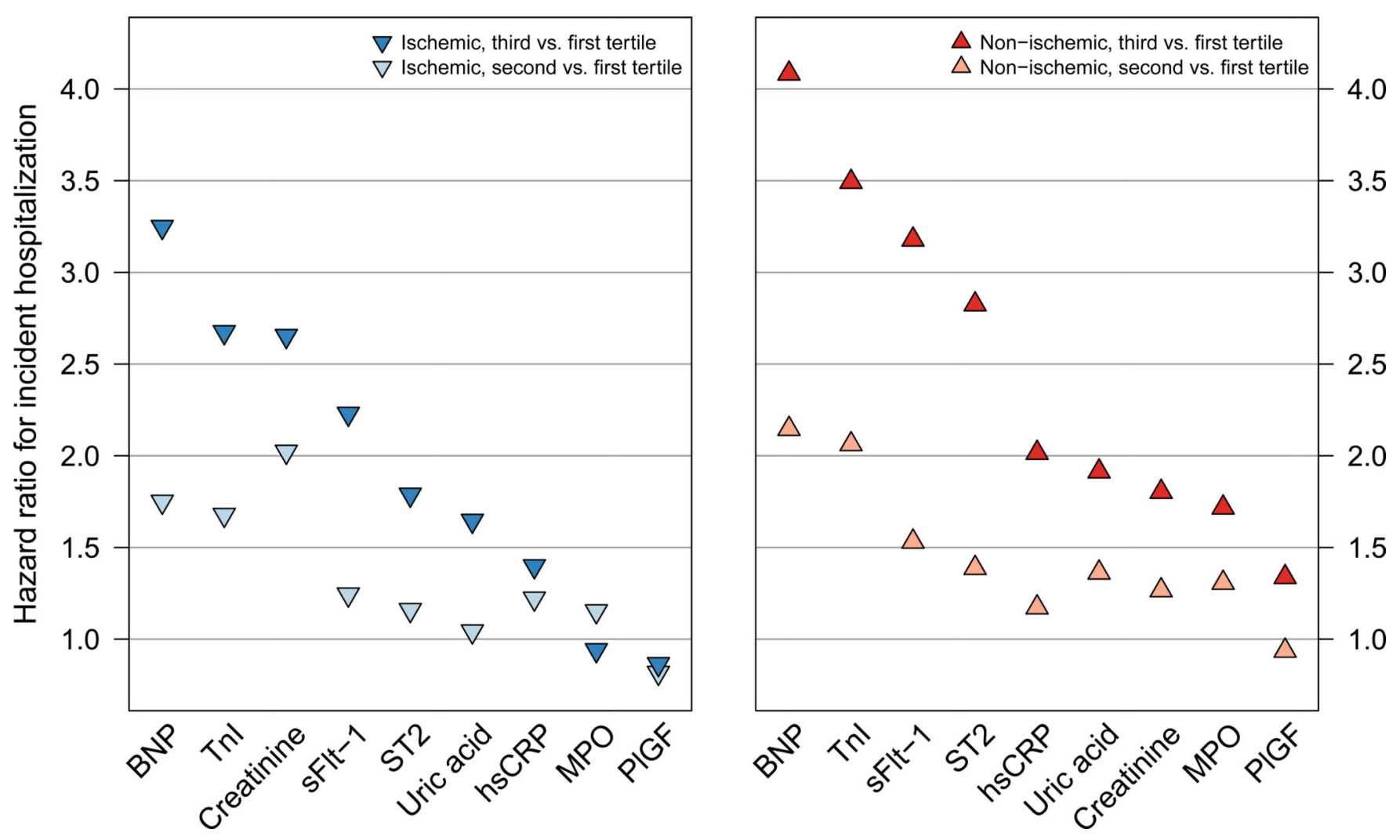

Figure 2.

Associations between biomarkers and risk of cardiac hospitalization according to second vs. first and third vs. first tertile for each biomarker distribution, among participants with ischemic and nonischemic heart failure etiology; estimates adjusted for age, gender, and race (Caucasian, African American, or other) 
Table 1

Characteristics of study participants at enrollment; summaries presented as n (\%) unless noted otherwise

\begin{tabular}{|c|c|c|c|c|}
\hline & $\begin{array}{l}\text { All participants } \\
n=1512\end{array}$ & $\begin{array}{l}\text { Ischemic } \\
\text { etiology } \\
\mathrm{n}=\mathbf{4 5 4}\end{array}$ & $\begin{array}{l}\text { Non-ischemic } \\
\text { etiology } \\
\mathrm{n}=1058\end{array}$ & $\mathbf{p}^{*}$ \\
\hline \multicolumn{5}{|l|}{ Demographic characteristics } \\
\hline Age, years, mean (SD) & $56(15)$ & $63(10)$ & $53(15)$ & $<0.001$ \\
\hline Male & $1001(66)$ & $375(83)$ & $626(59)$ & $<0.001$ \\
\hline Race & & & & $<0.001$ \\
\hline Caucasian & $1124(74)$ & $394(87)$ & $730(69)$ & \\
\hline African American & $332(22)$ & $41(9)$ & $291(28)$ & \\
\hline Other & $56(4)$ & $19(4)$ & $37(3)$ & \\
\hline \multicolumn{5}{|l|}{ Medical history and risk factors } \\
\hline History of hypertension & $883(58)$ & $299(66)$ & $584(55)$ & $<0.001$ \\
\hline History of diabetes & $443(29)$ & $196(43)$ & $247(23)$ & $<0.001$ \\
\hline Tobacco use & & & & $<0.001$ \\
\hline Current & $137(9)$ & $36(8)$ & 101(10) & \\
\hline Former & $808(54)$ & $317(70)$ & $491(47)$ & \\
\hline Never & $557(37)$ & $101(22)$ & $456(44)$ & \\
\hline Hypercholesterolemia & $753(50)$ & $319(70)$ & $434(41)$ & $<0.001$ \\
\hline \multicolumn{5}{|l|}{ Heart failure characteristics } \\
\hline NYHA functional classification & & & & $<0.001$ \\
\hline I & $258(17)$ & $48(11)$ & $210(20)$ & \\
\hline II & $691(46)$ & $198(44)$ & $493(47)$ & \\
\hline III & $446(30)$ & $160(35)$ & $286(27)$ & \\
\hline IV & $113(7)$ & $47(10)$ & $66(6)$ & \\
\hline Ischemic etiology & $454(30)$ & - & - & - \\
\hline Cardiac resynchronization therapy & $377(25)$ & $170(37)$ & $207(20)$ & $<0.001$ \\
\hline Defibrillator & $635(42)$ & $286(63)$ & $349(33)$ & $<0.001$ \\
\hline \multicolumn{5}{|l|}{ Medication use } \\
\hline ACE inhibitors or ARBs & $1316(87)$ & $403(89)$ & $913(86)$ & 0.22 \\
\hline Aldosterone antagonists $A$ & $508(34)$ & $189(42)$ & $319(30)$ & $<0.001$ \\
\hline Aspirin & $822(54)$ & $361(80)$ & $461(44)$ & $<0.001$ \\
\hline Beta-blockers & $1328(88)$ & $410(90)$ & $918(87)$ & 0.065 \\
\hline Digoxin & $586(39)$ & $228(50)$ & $358(34)$ & $<0.001$ \\
\hline Diuretics & $1184(78)$ & $389(86)$ & $795(75)$ & $<0.001$ \\
\hline HMG CoA reductase inhibitors & $753(50)$ & $361(80)$ & $392(37)$ & $<0.001$ \\
\hline \multicolumn{5}{|l|}{ Clinical measures, mean (SD) } \\
\hline Body mass index, $\mathrm{kg} / \mathrm{m}^{2}$ & $30(7.2)$ & $30(5.9)$ & $30(7.7)$ & 0.11 \\
\hline Systolic blood pressure, $\mathrm{mmHg}$ & $114(20)$ & 111(19) & $115(21)$ & $<0.001$ \\
\hline Diastolic blood pressure, $\mathrm{mmHg}$ & $70(12)$ & $68(11)$ & $71(12)$ & $<0.001$ \\
\hline eGFR, $\mathrm{ml} / \mathrm{min} / 1.73 \mathrm{~m}^{2}$ & $84(32)$ & $74(29)$ & $88(33)$ & $<0.001$ \\
\hline Sodium, $\mathrm{mEq} / \mathrm{L}$ & $139(3.4)$ & $139(3.8)$ & $139(3.2)$ & 0.009 \\
\hline
\end{tabular}




\begin{tabular}{lllll}
\hline & $\begin{array}{l}\text { All participants } \\
\text { n=1512 }\end{array}$ & $\begin{array}{l}\text { Ischemic } \\
\text { etiology } \\
\mathbf{n = 4 5 4}\end{array}$ & $\begin{array}{l}\text { Non-ischemic } \\
\text { etiology } \\
\text { n=1058 }\end{array}$ & $\mathbf{p}^{*}$ \\
\hline Ejection fraction, \% & $33(17)$ & $28(13)$ & $36(18)$ & $<0.001$ \\
Biomarkers, median (IQR) & & & & \\
BNP, pg/mL & $173(47,579)$ & $353(131,849)$ & $120(33,44)$ & $<0.001$ \\
PlGF, pg/mL & $18.7(15.1,22.9)$ & $21.2(17.5,25.3)$ & $17.6(14.4,21.7)$ & $<0.001$ \\
sFlt-1, pg/mL & $309(260,379)$ & $320(268,387)$ & $302(255,376)$ & 0.002 \\
hsCRP, mg/L & $0.35(0.14,0.90)$ & $0.40(0.16,1.04)$ & $0.33(0.14,0.82)$ & 0.018 \\
MPO, pmol/L & $137(95,227)$ & $138(96,232)$ & $137(95,224)$ & 0.65 \\
ST2, ng/mL & $27.4(20.0,40.5)$ & $31.8(22.4,48.7)$ & $25.4(19.0,37.3)$ & $<0.001$ \\
TnI & & & & $<0.001$ \\
Detectable, n (\%) & $975(64)$ & $371(82)$ & $604(57)$ & \\
TnI, ng/L, median (IQR) ${ }^{* *}$ & $20(10,40)$ & $20(10,30)$ & $20(10,40)$ & \\
Creatinine, mg/dL & $0.92(0.76,1.25)$ & $1.07(0.82,1.44)$ & $0.87(0.74,1.15)$ & $<0.001$ \\
Uric acid, mg/dL & $6.9(5.7,8.8)$ & $7.5(6.1,9.4)$ & $6.8(5.4,8.5)$ & $<0.001$ \\
\hline
\end{tabular}

* $\mathrm{P}$ values obtained from two-sample t-tests, Wilcoxon rank-sum tests, or chi-squared tests comparing characteristics between participants with ischemic and non-ischemic etiology

**

Among those participants with a detectable troponin I

SD, standard deviation; IQR, $25^{\text {th }}$ and $75^{\text {th }}$ percentile; NYHA, New York Heart Association; ACE, angiotensin-converting enzyme; ARBs, angiotensin receptor blockers; eGFR; estimated glomerular filtration rate; BNP, B-type natriuretic peptide; PlGF, placental growth factor; sFlt-1, soluble fms-like tyrosine kinase receptor-1; hsCRP, high-sensitivity C-reactive protein; MPO, myeloperoxidase; ST2, soluble toll-like receptor-2; TnI, troponin I 


\section{Table 2}

Associations between biomarkers and risk of cardiac hospitalization according to second vs. first and third vs. first tertile for each biomarker distribution

\begin{tabular}{|c|c|c|c|c|}
\hline & $\begin{array}{l}\text { Unadjusted model } \\
\text { HR }(95 \% \text { CI })\end{array}$ & $\mathbf{P}$ & $\begin{array}{l}\text { Adjusted Model } \\
\text { HR }(95 \% \text { CI })\end{array}$ & $\mathbf{P}$ \\
\hline \multicolumn{5}{|l|}{ BNP } \\
\hline $80-377$ vs. $<80 \mathrm{pg} / \mathrm{mL}$ & $2.0(1.7,2.4)$ & $<0.001$ & $2.0(1.6,2.6)$ & $<0.001$ \\
\hline$\geq 377$ vs. $<80 \mathrm{pg} / \mathrm{mL}$ & $3.8(3.2,4.5)$ & $<0.001$ & $3.8(2.9,4.9)$ & $<0.001$ \\
\hline \multicolumn{5}{|l|}{ TnI } \\
\hline $5-20$ vs. $<5 \mathrm{ng} / \mathrm{L}$ & $2.0(1.7,2.4)$ & $<0.001$ & $2.0(1.6,2.4)$ & $<0.001$ \\
\hline$\geq 20$ vs. $<5 \mathrm{ng} / \mathrm{L}$ & $3.4(2.9,4.1)$ & $<0.001$ & $3.3(2.8,3.9)$ & $<0.001$ \\
\hline \multicolumn{5}{|l|}{ sFlt-1 } \\
\hline $274-346$ vs. $<274 \mathrm{pg} / \mathrm{mL}$ & $1.5(1.2,1.7)$ & $<0.001$ & $1.4(1.2,1.7)$ & $<0.001$ \\
\hline$\geq 346$ vs. $<274 \mathrm{pg} / \mathrm{mL}$ & $3.0(2.5,3.6)$ & $<0.001$ & $2.8(2.4,3.4)$ & $<0.001$ \\
\hline \multicolumn{5}{|l|}{ ST2 } \\
\hline $22.4-35.0$ vs. $<22.4 \mathrm{ng} / \mathrm{mL}$ & $1.3(1.1,1.5)$ & 0.005 & $1.3(1.1,1.6)$ & 0.002 \\
\hline$\geq 35.0$ vs. $<22.4 \mathrm{ng} / \mathrm{mL}$ & $2.4(2.0,2.9)$ & $<0.001$ & $2.3(2.0,2.8)$ & $<0.001$ \\
\hline \multicolumn{5}{|l|}{ Creatinine } \\
\hline $0.80-1.11$ vs. $<0.80 \mathrm{mg} / \mathrm{dL}$ & $1.5(1.3,1.8)$ & $<0.001$ & $1.4(1.2,1.7)$ & $<0.001$ \\
\hline$\geq 1.11$ vs. $<0.80 \mathrm{mg} / \mathrm{dL}$ & $2.2(1.8,2.6)$ & $<0.001$ & $1.9(1.6,2.4)$ & $<0.001$ \\
\hline \multicolumn{5}{|l|}{ Uric acid } \\
\hline $6.1-8.1$ vs. $<6.1 \mathrm{mg} / \mathrm{dL}$ & $1.4(1.1,1.6)$ & $<0.001$ & $1.3(1.1,1.5)$ & 0.009 \\
\hline$\geq 8.1 \mathrm{vs} .<6.1 \mathrm{mg} / \mathrm{dL}$ & $2.0(1.7,2.4)$ & $<0.001$ & $1.9(1.6,2.2)$ & $<0.001$ \\
\hline \multicolumn{5}{|l|}{ hsCRP } \\
\hline $0.20-0.61$ vs. $<0.20 \mathrm{mg} / \mathrm{L}$ & $1.2(1.0,1.4)$ & 0.030 & $1.2(0.99,1.4)$ & 0.065 \\
\hline$\geq 0.61$ vs. $<0.20 \mathrm{mg} / \mathrm{L}$ & $1.9(1.6,2.3)$ & $<0.001$ & $1.8(1.5,2.1)$ & $<0.001$ \\
\hline \multicolumn{5}{|l|}{ MPO } \\
\hline $109-182$ vs. $<109 \mathrm{pmol} / \mathrm{L}$ & $1.2(1.0,1.5)$ & 0.016 & $1.2(1.0,1.5)$ & 0.022 \\
\hline$\geq 182$ vs. $<109 \mathrm{pmol} / \mathrm{L}$ & $1.4(1.2,1.7)$ & $<0.001$ & $1.4(1.2,1.7)$ & $<0.001$ \\
\hline \multicolumn{5}{|l|}{ PlGF } \\
\hline $16.2-21.3$ vs. $<16.2 \mathrm{pg} / \mathrm{mL}$ & $0.95(0.80,1.1)$ & 0.55 & $0.91(0.77,1.1)$ & 0.30 \\
\hline$\geq 21.3$ vs. $<16.2 \mathrm{pg} / \mathrm{mL}$ & $1.2(1.0,1.5)$ & 0.022 & $1.2(0.96,1.4)$ & 0.12 \\
\hline
\end{tabular}

* Adjusted model includes age, gender, race (Caucasian, African American, or other), and heart failure etiology (ischemic or non-ischemic)

HR, hazard ratio; CI, confidence interval; BNP, B-type natriuretic peptide; TnI, troponin I; sFlt-1, soluble fins-like tyrosine kinase receptor-1; ST2, soluble toll-like receptor-2; hsCRP, high-sensitivity C-reactive protein; MPO, myeloperoxidase; PlGF, placental growth factor 
Table 3

Unadjusted hazard ratios for incident cardiac hospitalization comparing groups defined by BNP (dichotomized at median) and troponin (dichotomized as detectable vs. not)

\begin{tabular}{lll}
\hline & $\begin{array}{l}\text { BNP } \\
<\mathbf{1 7 3} \mathbf{~ p g} / \mathbf{m L}\end{array}$ & $\begin{array}{l}\mathbf{1 7 3} \mathbf{~ p g} / \mathbf{m L} \\
\mathbf{N} ; \mathbf{H R}(\mathbf{9 5 \%} \mathbf{C I}) ; \mathbf{P}\end{array}$ \\
\hline TnI & $\mathbf{N}$ HR $(\mathbf{9 5 \%} \mathbf{C I}) ; \mathbf{P}$ & $103 ; 2.3(1.7,3.1) ;<0.001$ \\
\hline Not detectable & $434 ;$ Referent & $653 ; 4.1(3.4,4.9) ;<0.001$ \\
Detectable & $322 ; 2.3(1.8,2.8) ;<0.001$ & \\
\hline
\end{tabular}

$\mathrm{N}$, sample size in each group; HR, hazard ratio, CI, confidence interval; BNP, B-type natriuretic peptide; TnI, troponin I 